\title{
Forecasting Real Time Series Data using Deep Belief Net and Reinforcement Learning
}

\author{
Takaomi Hirata $^{1}$, Takashi Kuremoto ${ }^{2}$, Masanao Obayashi $^{2}$, Shingo Mabu $^{2}$, Kunikazu Kobayashi ${ }^{3}$ \\ ${ }^{1}$ Graduate School of Science and Engineering, Yamaguchi University, Tokiwadai 2-16-1, \\ Ube, Yamaguchi 755-8611, Japan \\ ${ }^{2}$ Graduate School of Science and Technology for Innovation, Yamaguchi University, Tokiwadai 2-16-1, \\ Ube, Yamaguchi 755-8611, Japan \\ ${ }^{3}$ School of Information Science and Technology, Aichi Prefectural University, 1522-3 Ibaragabasama, \\ Nagakute, Aichi 480-1198, Japan \\ E-mail: \{v003we,wu,m.obayas,mabu\}@yamaguchi-u.ac.jp, kobayashi@ist.aichi-pu.ac.jp
}

\begin{abstract}
Hinton's deep auto-encoder (DAE) with multiple restricted Boltzmann machines (RBMs) is trained by the unsupervised learning of RBMs and fine-tuned by the supervised learning with error-backpropagation (BP). Kuremoto et al. proposed a deep belief network (DBN) with RBMs as a time series predictor, and used the same training methods as DAE. Recently, Hirata et al. proposed to fine-tune the DBN with a reinforcement learning (RL) algorithm named "Stochastic Gradient Ascent (SGA)" proposed by Kimura \& Kobayashi and showed the priority to the conventional training method by a benchmark time series data CATS. In this paper, DBN with SGA is invested its effectiveness for real time series data. Experiments using atmospheric CO2 concentration, sunspot number, and Darwin sea level pressures were reported.
\end{abstract}

Keywords: deep learning, restricted Boltzmann machine, stochastic gradient ascent, reinforcement learning, errorbackpropagation

\section{Introduction}

Deep learning (DL) is the novel kernel technique of artificial intelligence (AI) developed rapidly in nowadays. As the training method of artificial neural networks (ANNs), in 2006, DL firstly is introduced by Hinton's deep auto-encoder (DAE) [1], which has multiple stacked restricted Boltzmann machines (RBMs). The learning process of DAE is divided into two phases: firstly, pretraining, which is a kind of unsupervised learning using the gradient of network energy of RBMs, and secondly fine-tuning using the supervised learning: error-backpropagation (BP) [2].

To deal with the adaptive behavior acquisition problem in unknown environment, reinforcement learning (RL), which is a kind of machine learning algorithm adjusting the output of an agent or system (learner) utilizing the rewards/punishment from the environment. , has been studied for decades [3] [4]. Recently, RL is also introduced into deep neural networks (DNNs)[5]-[7]. In [5], a deep Q-network (DQN) is proposed and applied to game (named ATARI) control and reached human level. In [6], a computer software named AlphaGo, using a DNN and RL, won the world champion of the game Go. In [7], we adopted a policy gradient RL algorithm [8] [9] into a deep belief net (DBN) proposed by Kuremoto et al. [10]-[13] instead of the conventional fine-tuning method BP. And using a benchmark data CATS which was used by time series forecasting competition with ANNs [14] [15], the DBN with RL showed the highest prediction precision comparing to all conventional methods 
utilized in the competition and the conventional DBN with BP learning [12] [13].

In this paper, we concentrate to investigate the effectiveness of the DBN with RL for real time series forecasting. Three kinds of real time series data which are weekly average of $\mathrm{CO} 2$ concentration in atmosphere at Hawaii, monthly average of sea level pressures at Darwin, the number of sunspot monthly provided by Aalto University [16] were used in the forecasting experiments, and the prediction precision was compared to the conventional BP learning method. And as the results, DBN with RL showed the higher performance than the conventional DBN with BP in the process of fine-tuning of the network for these real data forecasting.

\subsection{DBN with RBMs and MLP}

As a neural predictor model, a DBN composed by multiple RBMs and a MLP is shown in Fig. 1 [7] [12] [13]. The visible layer of RBM 1 ( $1^{\text {st }}$ Layer) are input with raw data of time series data (omitted in the figure). The hidden layer of RBM $L+1$ are used as the input layer of the MLP, where $l=1,2, \ldots, L$. The output of MLP is with one unit in the case of DBN using BP learning, and it has two units which are parameters of Gaussian distribution function used in the case of SGA learning method [4] [7].

\subsection{BP learning for $D B N$}

Let $E$ is the mean squared error (MSE) between the

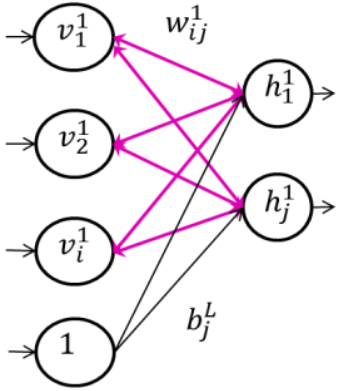

RBM 1(1st Layer)

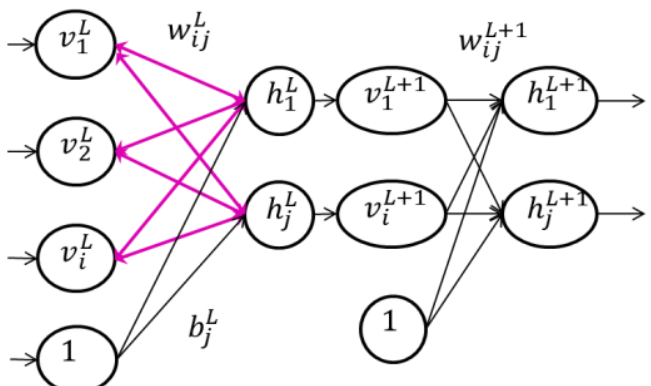

RBM L(L-th Layer)

RBM L+1( L+1-th Layer)

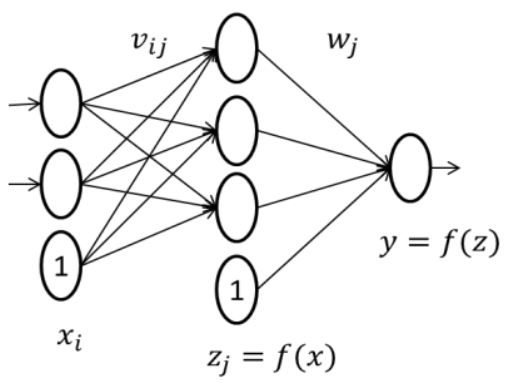

MLP

Fig. 1. A structure of DBN composed by RBMs and a MLP

\section{DBN with BP}

In [10] [11], Kuremoto et al. firstly applied Hinton \& Slakhutdinov's deep belief net (DBN) with restricted Boltzmann machines (RBMs) to the field of time series forecasting. In [12] and [13], Kuremoto, Hirata, et al. constructed a DBN with RBMs and a multi-layer perceptron (MLP) to improve the previous time series predictor with RBMs. In [7], Hirata et al. adopted a RL method named "stochastic gradient ascent" (SGA) proposed by Kimura \& Kobayashi [8] [9] into DBN instead of the BP learning used in the fine-tuning of the network. In this section, the structure of DBN and learning methods are introduced. output of DBN and the teacher signal, the weight of connections $w$ between layers of RBMs and MLP, and the bias of RBMs $b$ are modified as following.

$$
\begin{aligned}
w_{i j}^{l^{\text {new }}} & =w_{i j}^{l^{\text {old }}}+\alpha \frac{\partial E}{\partial w_{i j}^{l^{\text {old }}}} \\
& =w_{i j}^{l^{\text {old }}}+\alpha\left(\sum_{j} \frac{\partial E}{\partial w_{i j}^{l+2^{\text {old }}}} w_{i j}^{l+1^{\text {old }}}\right) \cdot\left(1-h_{j}^{l}\right) \cdot v_{i}^{l}
\end{aligned}
$$




$$
\begin{aligned}
b_{j}^{l_{\text {new }}}= & b_{j}^{l^{\text {old }}}+\alpha \frac{\partial E}{\partial b_{j}^{\text {lold }}} \\
& =b_{j}^{l^{\text {old }}}+\alpha\left(\sum_{j} \frac{\partial E}{\partial b_{j}^{l+2^{\text {old }}}} b_{j}^{l+1^{\text {old }}}\right) \cdot\left(1-h_{j}^{l}\right)
\end{aligned}
$$

where $a$ is the learning rate, $i, j \in\{1,2, \ldots\}$ indicate the number of units in layers of RBM and MLP, 1 is the number of layers of DBN.
Kobayashi suggested to adopt the inertia of $\mathrm{CE}$ and named the learning method as "SGA" and applied it to control problem in the continuous space [8].

$$
\begin{aligned}
& \overline{D_{i}}(t)=e_{i}(t)+\gamma \overline{D_{i}}(t-1) \\
& \Delta \varpi_{i}(t)=\left(r_{t}-b\right) \overline{D_{i}}(t)
\end{aligned}
$$

In [4], Kuremoto et al. proposed to adopt SGA for time series forecasting where reward was given as following.

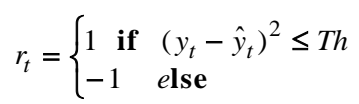

\begin{tabular}{|l|l|l|l|l|}
\hline \multicolumn{1}{|c|}{ Data Series } & \multicolumn{1}{|c|}{ Total size } & \multicolumn{1}{c|}{ Testing size } & $\begin{array}{l}\text { DBN with BP } \\
\text { (The number of units in } \\
\text { different layers) }\end{array}$ & $\begin{array}{l}\text { DBN with SGA } \\
\text { (The number of units } \\
\text { in different layers) }\end{array}$ \\
\hline $\mathrm{CO} 2$ & 2225 & 225 & $15-17-17-1$ & $20-18-7-2$ \\
\hline Sea level pressure & 1400 & 400 & $16-18-18-1$ & $16-20-8-7-2$ \\
\hline Sun spot number & 3078 & 578 & $20-20-17-18-1$ & $19-19-20-10-2$ \\
\hline
\end{tabular}

Table 1 Sizes of time series data and structures of prediction networks

\subsection{SGA learning for $D B N$}

The SGA algorithm and the learning rule for the weight of DBN's layers and parameters of the stochastic policy (Gaussian distribution function) were introduced in [7]. SGA is an improved RL algorithm of William's "REINFORCE", i.e., Reward Increment $=$ Nonnegative Factor $\times$ Offset Reinforcement $\times$ Characteristic Eligiblilty:

$$
\begin{gathered}
\Delta w_{i}=\alpha_{i}\left(r-b_{i}\right) e_{i} \\
e_{i}=\frac{\partial \ln g(\xi, \mathbf{w}, \mathbf{x})}{\partial w_{i}} \\
g(\xi, \mathbf{w}, \mathbf{x})=\operatorname{Pr}(y=\xi \mid \mathbf{w}, \mathbf{x}) \\
y=f\left(\sum_{j} w_{i j} x_{j}\right)
\end{gathered}
$$

where $\xi, \mathbf{w}, \mathbf{x}, \alpha_{i}, r, b_{i}, e_{i}, y$ are the stochastic output, the connection weight to unit $i$, the input to unit $i$, learning rate, reward, bias, and Characteristic Eligibility (CE), the output of network, respectively [9]. Kimura \& where $y_{t}, \hat{y}_{t}$, Th are the teach signal, the output of network, threshold, respectively.

In [7], Hirata et al. suggested to use a variable threshold according to the change of prediction error instead of the fixed threshold in Eq. (9).

$$
T h \equiv \varepsilon_{t}=\beta \mathbf{M S E}
$$

where $\beta$, MSE are constant and mean squared error of prediction of DBN.

\section{Prediction Experiments and Results}

We predicted three types of natural phenomenon time series data given by Aalto University [16].

$>\mathrm{CO} 2$ : Atmospheric $\mathrm{CO} 2$ from continuous air samples Weekly averages atmospheric $\mathrm{CO} 2$ concentration derived from continuous air samples, Hawaii, 2225 values

Sea level pressures: Monthly values of the Darwin Sea Level Pressure series, 1882-1998, 1300 values 
Sunspot Number: Monthly averages of sunspot numbers from 1749 through the present, 3078 values $>$

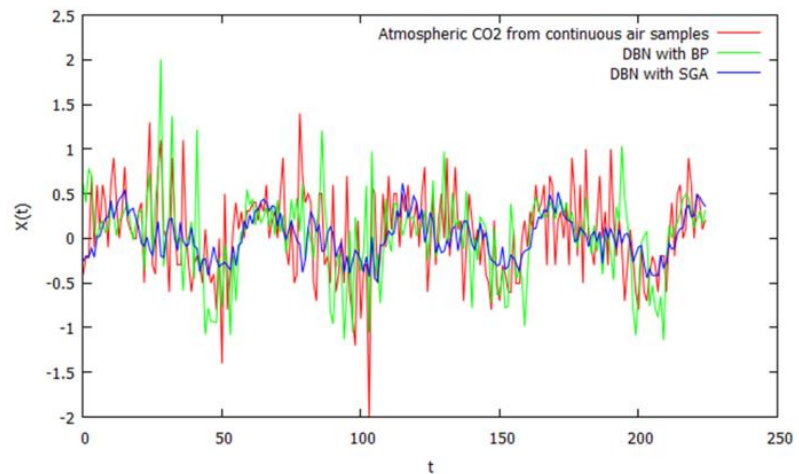

Fig. 2. Prediction result of $\mathrm{CO} 2$ data

In Table 1, the number of samples and structures of different DBNs were listed. To decide the number of RBMs, and the number of units on different layers of RBMs and MLP, random search (RS) [17] was used in the experiments. As an optimization method, RS used random values of parameter vector spaces to find the lower forecasting error (MSE).

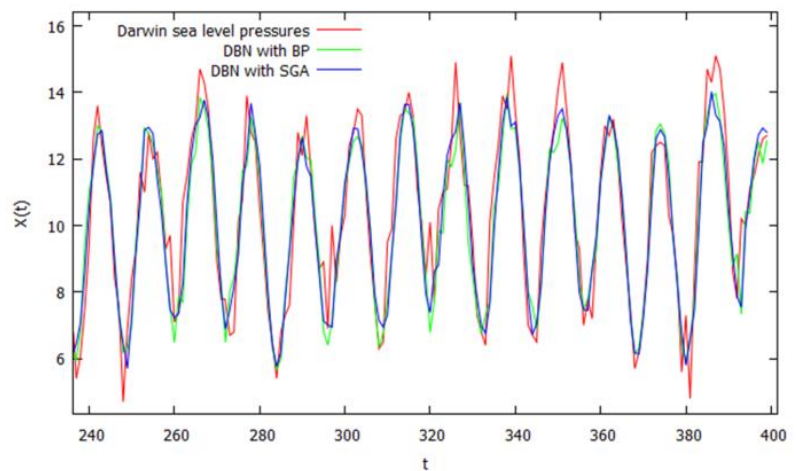

Fig. 3. Prediction result of Sea level pressure data

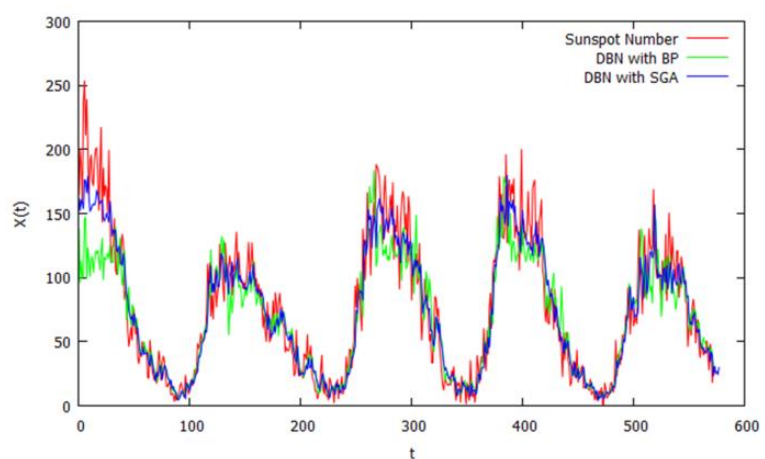

Fig.4. Prediction result of Sun spot number data
Table 2 Pdiction MSE of real time series data

\begin{tabular}{|c|c|c|}
\hline & $\begin{array}{c}\text { DBN with } \\
\text { BP }\end{array}$ & $\begin{array}{c}\text { DBN with } \\
\text { SGA }\end{array}$ \\
\hline CO2 & 0.2671 & $\mathbf{0 . 2 0 4 7}$ \\
\hline Sea level pressure & 0.9902 & $\mathbf{0 . 9 0 0 3}$ \\
\hline Sun spot number & 733.51 & $\mathbf{3 6 4 . 0 5}$ \\
\hline
\end{tabular}

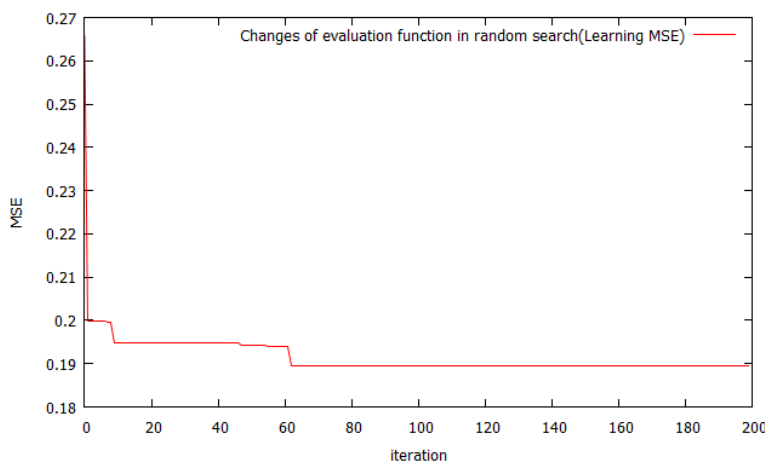

Fig.5. Changes of evaluation function in random search (CO2, DBN with SGA)

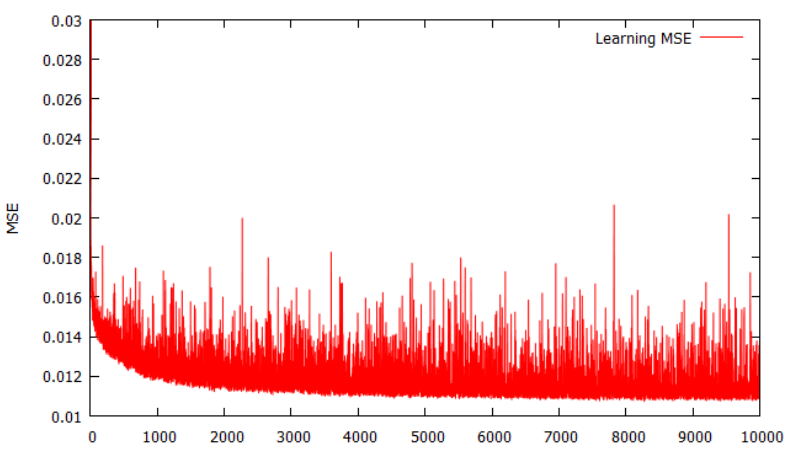

Fig.6. Changes in learning MSE (CO2, DBN with SGA)

In Fig. 2 to Fig. 4, the one-ahead prediction results of DBN with BP and DBN with SGA were shown. In Table 2, the comparison of forecasting precision (MSE) of these different learning methods for DBN was given. The DBN with SGA showed its priority to the DBN with BP in all cases of real time series data.

The change of evaluation function in the case of DBN with SGA for CO2 forecasting is shown in Fig. 5 as a sample. In Fig. 6, the change of MSE in SGA learning process is shown. The vibration of MSE in Fig. 6 needs to be reduced by optimizing the value of learning rate and reward, it remains as the future work. 


\section{Conclusion}

In this paper, a reinforcement learning (RL) method "stochastic gradient ascent (SGA)" for fine-tuning of a deep belief net (DBN) with multiple restricted Boltzmann machines (RBMs) and a multi-layer perceptron (MLP) was compared to the conventional learning method error backpropagation (BP) of DBN for real time series data forecasting. Different from the supervised learning method which uses learning error exhaustively, RL used a reward function which allowed a range of errors between the output of the model and the teach signal and it raised the forecasting precision for the real time series data as shown as results of experiments.

\section{Acknowledgements}

This work was supported by JSPS KAKENHI Grant No. 26330254 and No.25330287.

\section{References}

1. G.E. Hinton, R.R. Salakhutdinov, Reducing the dimensionality of data with neural networks, Science, Vol.313, 2006, pp. 504-507.

2. D. E. Rumelhart, G. E. Hinton, \& R.J. Williams, Learning representation by back-propagating errors, Nature, Vol. 232, No. 9, 1986, pp. 533-536.

3. R.S. Sutton, A.G. Barto: Reinforcement Learning: An Introduction, 1998, The MIT Press

4. T. Kuremoto, M. Obayashi, M. Kobayashi, Neural Forecasting Systems. In Reinforcement Learning, Theory and Applications, pp. 1-20, 2008, INTECH.

5. V. Mnih, et al., Human-level control through deep reinforcement learning, Nature, Vol. 518, 2015, pp. 529533

6. D. Silver, et al., Mastering the game of Go with deep Neural networks and tree search, Nature, 529, 2016, pp. 484-489

7. T. Hirata, T. Kuremoto, M. Obayashi, S. Mabu, K. Kobayashi, Deep Belief Network Using Reinforcement Learning and Its Applications to Time Series Forecasting, Proceedings of Neural Information Processing: The 23rd International Conference (ICONIP 2016), Vol.3, 2016, pp. $30-37$

8. H. Kimura, S. Kobayashi, Reinforcement learning for continuous action using stochastic gradient ascent, Proceedings of The 5th Intelligent Autonomous Systems, 1998, pp. 288-295.

9. R.J. Williams: Simple statistical gradient-following for connectionist reinforcement learning, Machine Learning, Vol. 8, 1992, pp. 229-256
10. T. Kuremoto, S. Kimura, K. Kobayashi, M. Obayashi, Time series forecasting using restricted Boltzmann machines, Proceedings of The $8^{\text {th }}$ International Conference on Intelligent Computing (ICIC 2012), 2012, pp.17-22.

11. T. Kuremoto, S. Kimura, K. Kobayashi, M. Obayashi, Time series forecasting using a deep belief network with restricted Boltzmann machines, Neurocomputing, Vol.137, No.5, 2014, pp.47-56.

12. T. Kuremoto, T. Hirata, M. Obayashi, S. Mabu, K. Kobayashi, Forecast Chaotic Time Series Data by DBNs, Proceedings of The 7th International Congress on Image and Signal Processing (CISP 2014), 2014, pp. 1304-1309.

13. T. Hirata, T. Kuremoto, M. Obayashi, S. Mabu, Time Series Prediction Using DBN and ARIMA, Proceedings of The International Conference on Computer Application Technologies (CCATS 2015), 2015, pp.2429.

14. A. Lendasse, E. Oja, O. Simula, M. Verleysen, Time Series Prediction Competition: The CATS Benchmark, Proceedings of The International Joint Conference on Neural Networks (IJCNN'04), 2004, pp1615-1620.

15. A. Lendasse, E. Oja, O. Simula, M. Verleysen, Time Series Prediction Competition: The CATS Benchmark, Neurocomputing, Vol. 70, 2007, pp.2325-2329.

16. AML research group, The Aalto University School of Science: http://research.ics.aalto.fi/eiml/datasets.shtml

17. J. Bergstra, Y. Bengio, Random Search for HyperParameter Optimization, Journal of Machine Learning Research, Vol. 13, 2012, pp.281-305. 